\title{
The beneficial and deleterious role of dietary polyphenols on chronic degenerative diseases by regulating gene expression
}

\author{
Guojing Qu ${ }^{1}$, Jinhua Chen ${ }^{2}$, Xiuli Guo ${ }^{2, *}$ \\ ${ }^{1}$ Shandong University Taishan College (Biological Research Training Program for Top-notch Students), Ji'nan, Shandong, China; \\ ${ }^{2}$ Department of Pharmacology, School of Pharmaceutical Sciences, Shandong University, Jinan, Shandong, China.
}

\begin{abstract}
Summary Dietary polyphenols, a natural component in many kinds of foods such as fruits and vegetables, play essential roles in a wide range of plant functions. Importantly, the discovery of the functions of polyphenols including anti-oxidant, anti-carcinogenic and anti-inflammatory has been appealing to researchers' attentions. Dietary polyphenols have shown protective effects on chronic degenerative diseases (CDD) such as cardiovascular diseases, cancers, and neurodegenerative diseases by regulating gene expression. Dietary polyphenols also affect the composition and activity of gut microbiota, in reverse, gut microbiota influences the bioavailability and physiological activity of dietary polyphenols. However, not all kinds of dietary polyphenols are beneficial for human health. The potential deleterious effects of several dietary polyphenols have been reported by inducing DNA damage and gene mutants. This review summarizes the potential therapeutic effects of dietary polyphenols on chronic degeneration diseases, the polyphenols-gut microbiota interactions, and the potential dangers of individual dietary polyphenols on human health.
\end{abstract}

Keywords: Dietary polyphenols, chronic degenerative diseases, expression regulation, therapeutic effects, deleterious effects

\section{Introduction}

Dietary polyphenols are common secondary metabolites in various plant foods, which derive from L-phenylalanine and play an important role in the normal growth and functions of the plants (1). They are available for the pigmentation of flower, fruit and vegetables to adapt to use bees or moths as pollinators and seed dispersers. They protect plants against ultraviolet light, act as the plant defense against pathogenic microorganisms, for example, phenolic monoterpenes (such as carvacrol, thymol, etc.) have antifungal activity against plant pathogenic fungi $(2,3)$. Recently, human epidemiological studies have shown that there is a positive correlation between the intake of polyphenols and the incidence of many chronic

Released online in J-STAGE as advance publication December 28, 2018.

*Address correspondence to:

Dr. Xiuli Guo, Department of Pharmacology, School of Pharmaceutical Sciences, Shandong University, No. 44, WenHuaXi Road, Ji'nan 250012, P.R. China.

E-mail: guoxl@sdu.edu.cn degenerative diseases, such as cardiovascular diseases, cancers, neurodegenerative diseases and diabetes $(4,5)$. One typical case is "French Paradox phenomenon" which is referred to as a relatively low incidence of coronary heart disease (CHD) in the French population, despite consuming a diet rich in saturated fat $(6)$. The possible explanation for the lower CHD in the French is the large consumption of wines, which are rich in some polyphenols. Another typical case is "Mediterranean diet" which was characterized by high consumption of olive oil, unrefined cereals, vegetables, fruits; moderate consumption of fish, cheese and wine, which was rich in dietary polyphenols. The health benefits and protective effects of "Mediterranean diet" against agerelated cognitive decline and cognitive impairment of Alzheimer's disease (AD) and vascular dementia (VaD) were well documented $(7,8)$. Therefore, there has been growing scientific interest in various chemical properties and biological effects of dietary polyphenols on chronic degenerative diseases.

More than 8,000 different polyphenols have been identified in edible plants. Their diverse chemical structures contribute to their multiple functions including anti-oxidative, anti-inflammatory, anti-carcinogenic, 
cardioprotective actions and anti-platelet properties, etc. $(9,10)$. However, the structure-activity relationship of dietary polyphenols is still unclear. Importantly, dietary polyphenols are found to bring potential danger to human health (11). Some dietary polyphenols have mutagenic and/or pro-oxidant effects, as well as interference with endogenous essential biochemical pathways (1214). Therefore, this review will summarize the recent understanding of the structure and structure-activity relationship, the therapeutic effects and molecular mechanism of dietary polyphenols on human diseases, and the polyphenols-gut microbiota interactions as well as the potential dangers of individual dietary polyphenols.

\section{The molecular structure and structure-activity relationship of dietary polyphenols}

Chemically, dietary polyphenols consist of one or more hydroxyl groups directly attached to a benzene ring. They can be classified according to their structure as flavonoids, phenolic acid derivatives, and the less common stilbenes or lignans in Table 1, and most percentages exist in the form of flavonoids $(4,15)$. Flavonoids have a common C6-C3-C6 structure consisting of two aromatic rings linked by a three carbon chain, and are usually organized as an oxygenated heterocycle in Figure 1. Among non-flavoniods polyphenols (Figure 2), stilbenes have a common C6C2-C6 structure consisting of two aromatic rings linked through a two carbon bridge with a double bond in Figure 2B (16). One of Stilbenes, resveratrol, has gotten more attention because of its multiple biological functions. Lignans consist of mostly two phenylpropanoid moieties connected via their side chain C8 carbons (17). They can be converted to enterodiol and enterolactone by intestinal bacteria in Figure 2C.

On the basis of the structural discrepancies, the structure-activity relationships of polyphenols have been established in vitro and in vivo (Table 2). Several catechins have shown their ability to induce apoptosis in human cancer cells (18). It has been found that catechins

Table 1. The categories of the representative polyphenolic compounds

\begin{tabular}{|c|c|}
\hline Categories & Representative compounds \\
\hline \multicolumn{2}{|l|}{ 1. Flavonoids } \\
\hline Flavanols & $\begin{array}{l}\text { (-)-Epigallocatechin-3-gallate (EGCG); } \\
\text { Epigallocatechin (EGC); (+)-Catechin. }\end{array}$ \\
\hline Flavonols & Myricetin; Quercetin. \\
\hline Flavones & Apigenin; Luteolin; Chrysin. \\
\hline Isoflavones & Genistein; Daidzein; Glycitein. \\
\hline Flavanones & Hesperetin; Naringenin. \\
\hline Anthocyanins & Anthocyanidins; Procyanidine. \\
\hline 2. Phenolic acids derivatives & Caffeic acid \\
\hline 3. Stilbenes & Resveratrol; Piceatannol. \\
\hline 4. Lignans & Secoisolariciresinol; Matairesinol. \\
\hline 5. Phenolic monoterpenes & Carvacrol, Thymol \\
\hline
\end{tabular}

with a pyrogallol-type structure in a B-ring induced apoptosis and a 3-O-gallate group in a cis-relationship to the $\mathrm{B}$ ring enhanced the activity, whereas catechins without a pyrogallol-type structure in a molecule lacked this activity. (-)-epigallocatechin-3-gallate (EGCG) and its metabolite products, such as pyrogallol, gallic acid or quinones also have been reported to have important roles in inducing apoptosis (19). By investigating the molecular structure-affinity relationship of natural polyphenols for $\gamma$-globulin, Xiao et al. demonstrated that galloylated catechins and catechol-type catechins exhibited higher binding affinities for $\gamma$-globulin than non-galloylated and pyrogallol-type catechins (20). They also found that the glycosylation of resveratrol

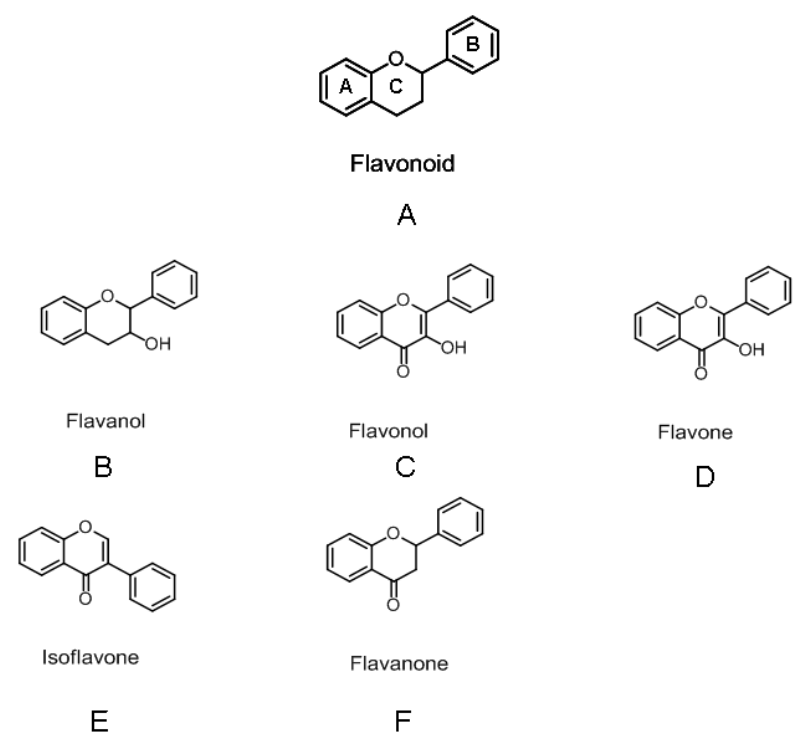

Figure 1. The chemical structure of different classes of Flavonoids. (A) The basic skeleton of flavonoid; (B) Flavanol; (C) Flavonol; (D) Flavone; (E) Isoflavone; (F) Flavanone.<smiles>O=C(O)/C=C/c1ccc(O)c(O)c1</smiles>

caffeic acid

A<smiles>C(=C/c1ccccc1)\c1ccccc1</smiles>

stilbene<smiles>Oc1ccc(C=Cc2cc(O)cc(O)c2)cc1</smiles>

resveratrol

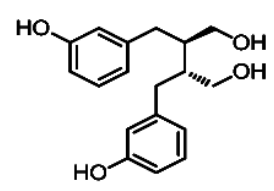

Enterodiol

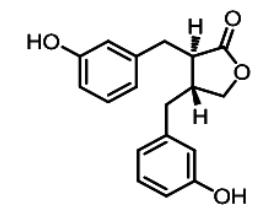

Enterolactone
C

Figure 2. The chemical structure of non-flavoniods polyphenols. (A) Caffeic acid (phenolic acids); (B) The basic skeleton of stilbene and resveratrol (stilbenes); (C) Enterodiol and enterolactone (lignans). 
Table 2. Structure-activity relationship of dietary polyphenols

\begin{tabular}{|c|c|c|}
\hline Polyphenol compounds & Group in structure & Activity \\
\hline \multirow[t]{4}{*}{ Flavonoids } & Methoxy groups & Decrease antioxidation \\
\hline & Multiple hydroxyl groups & $\begin{array}{l}\text { Inhibition on lipid peroxidation and ROS, chelating } \\
\text { redox-active metals }\end{array}$ \\
\hline & A double bond and carbonyl in the heterocycle & Increase free radical scavenging activity \\
\hline & R3'-OH and R4'-OH substitutions & Inhibit MMP-9 activity \\
\hline \multirow[t]{4}{*}{ Catechins } & pyrogallol-type structure in B ring & Apoptosis induction \\
\hline & Without pyrogallol-type structure in B ring & Lack of apoptosis induction \\
\hline & 3-O-gallate group in cis- relationship to the B ring in catechins & Increase apoptosis induction \\
\hline & Galloylated catechins and Catechol-type catechins & $\begin{array}{l}\text { Higher affinity to } \gamma \text {-globulin than non-galloylated } \\
\text { or pyrogallol-type }\end{array}$ \\
\hline Isoflavones & 4'-OH and 7'-OH site of daidzein & Increase hydroxyl radical scavenging activity \\
\hline Anthocyanins & 3-OH group & $\begin{array}{l}\text { Better antiradical and reductant activities than trolox } \\
\text { and catechol }\end{array}$ \\
\hline Stibenes & Glycosylation of resveratrol & Decreased affinity to $\gamma$-globulin \\
\hline Lignans & Without ortho-dihydroxy group & Weak radical scavenging activity \\
\hline \multirow[t]{2}{*}{ Phenolic monoterpenes } & Phenolic hydroxyl and monoterpene in carvacrol or thymol & Antifungal activity \\
\hline & Ester derivatives of carvacrol or thymol & Increased antifungal activity \\
\hline
\end{tabular}

decreased its affinity for $\gamma$-globulin. Moreover, cardioprotective effects of flavonoids are mainly due to the multiple hydroxyl groups in their molecules. Multiple hydroxyl groups conferred upon the molecule inhibition of lipid peroxidation, chelating redox-active metals and attenuating other processes involving reactive oxygen species. Methoxy groups in flavonoids introduce unfavorable steric effects and increase the lipophilicity and membrane partitioning, which result in decreased antioxidation. A double bond and carbonyl function in the heterocycle or polymerization of the nuclear structure increases free radical scavenging activity by affording a more stable flavonoid radical through conjugation and electron delocalization (21). Daidzein, a soy isoflavone, possessed potent hydroxyl radical scavenging activity through forming stable daidzein radicals with highly reactive hydroxyl radicals by a hydrogen abstraction reaction with both $\mathrm{OH}$ functional groups, the 4'-OH and 7'-OH site of daidzein. The high enthalpic stabilization involved in daidzein radical formation at the 4 '-OH site can be partly attributed to better solvation through hydrogen-bonding interactions with water and higher electron density delocalization of the radical over the adjacent aromatic ring (22). Both anthocyanidins and anthocyanins showed better antiradical and reductant activities than trolox and catechol. A structure-activity relationship study showed that, the 3-OH group improved hydrogen atom donation because of the stabilization by anthocyanidins semiquinone-like resonance, while radicals of the 4,5 or 7-OH groups could only be stabilized by resonance through pyrylium oxygen in the presence of the $3-\mathrm{OH}$ group. The 3-OH group also enhanced electron donation (23). Furthermore, Saragusti et al. have demonstrated that flavonoid R3'-OH and R4'-OH substitutions were relevant to the inhibitory property against the activity of matrix metalloproteinase 9 (MMP-9), which plays an important role in the turnover of basement membrane type IV collagen during the formation of atherosclerotic plaques (24). However, Cai et al. reported that although lignans had hydroxyl groups, their radical scavenging activity was very weak. Lack of the ortho-dihydroxy structure was the main reason (25). In addition, the antifungal activity of phenolic monoterpenes (carvacrol, thymol) was related to the basic structures of phenolic hydroxyl and monoterpene, while the position of phenolic hydroxyl has no significant effect. Moreover, the ester derivatives of carvacrol or thymol showed stronger antifungal activity against pathogenic fungi than their parent structure (2).

\section{The beneficial effects of polyphenols on several chronic degenerative diseases}

Numerous action mechanisms of polyphenols including anti-oxidation, free radical scavenging, mitochondrial protection and anti-cancerization, regulation on transcription factors and membrane receptors have been investigated. Some research demonstrated that dietary polyphenols have important potential for effective treatment of chronic degenerative diseases (CDD) like cardiovascular diseases (CVD), cancers and neurodegenerative diseases (4).

\subsection{Dietary polyphenols and cardiovascular diseases}

Dietary polyphenols are beneficial in many cardiovascular diseases, including myocardial ischemia/ reperfusion, platelet aggregation, inflammation and atherosclerosis (26). Among numerous plausible 
mechanisms, which may be involved in cardiovascular protection, improvement of endothelial function and inhibition of angiogenesis in blood vessels has been shown. Yang et al. reported that resveratrol, an antioxidant existing in red wine, was effective in preventing myocardial ischemia/reperfusion injury which may be due to its antioxidant activity and upregulation of vascular endothelial growth factor B (27). EGCG, a bioactive ingredient of green tea, plays a protective role in the cardiovascular system by inhibiting the expression of angiotensin II type 1 receptor (AT-1R) and extracellular regulated protein kinases 1/2 (ERK1/2) and p38 mitogen-activated protein kinase (MAPK) signals, resulting in a decrease of proliferation of human vascular smooth muscle cells (HVSMCs) induced by homocysteine (Hcy) (28). Furthermore, dietary polyphenols have positive effects on vascular function and platelet function in humans to attenuate thrombosis. The consumption of polyphenol rich foods might impart anti-thrombotic and cardiovascular protective effects via their inhibition of platelet hyperactivation or aggregation. Polyphenols also alleviated fibrinogen binding to platelet surface (GPIIb-IIIa) receptors to reduce platelet recruitment for aggregation and inhibit platelet degranulation by targeting various additional platelet activation pathways (e.g. by blocking plateletADP, collagen receptors) (29). Khan et al. demonstrated that flavonoids were responsible for several healthpromoting properties, such as outstanding preclinical antiplatelet effects, and provided an ideal approach as templates for new, clinically effective and safe antiplatelet agents (30). Omega-3 polyunsaturated fatty acids (PUFA) rich Mediterranean diet has protective effects for CVD by reducing the risk for sudden death induced by cardiac arrhythmias and could treat hyperlipidemia and hypertension. Foods with omega-3 PUFA, purple grape juice (PGJ), and wine all reduce platelet aggregation and $\mathrm{P}$-selectin expression (31). PGJ suppressed platelet-mediated thrombosis by decreasing platelet aggregation and superoxide production, increasing platelet-derived NO release which represented a potential mechanism for the beneficial effects of PGJ in CVD. An abundance of new evidence points to inflammation as a major participant in the pathogenesis and development of cardiovascular diseases. Polyphenols have been reported to directly inhibit inflammatory responses of the innate immune system, such as nuclear factor-kappa B (NF- $\kappa \mathrm{B})$, and activate anti-inflammatory gene transcription factors, such as peroxisome proliferators-activated receptors- $\gamma$ (PPAR- $\gamma$ ), adenosine monophosphate activated protein kinase (AMPK), etc. to exert a protective effect against hypertension, atherosclerosis, dyslipidemias (32). The impact of polyphenols on the same molecular targets as pharmacological interventions on inflammation makes it possible to develop a unique, non-pharmacological approach for CVD treatment.

\subsection{Dietary polyphenols and cancers}

Though great progress has been made in cancer therapy in the past several decades, the incidence and mortality of cancer has been increasing endlessly. By epidemiologic investigation, polyphenols-rich diets have been shown to be associated with a lower risk of cancers. Therefore, numerous studies have been conducted to explore the beneficial effects of polyphenols on various cancers. Green tea polyphenols defend healthy cells from malignant transformation and induce apoptosis locally in oral cancer cells (33). Polyphenols extracted from both green tea and ginger showed antiproliferative and apoptosis-mediated cytotoxic effects on human non-small lung cancer cells (34). Polyphenols and sterols extracted from virgin argan oil have also shown antiproliferative and pro-apoptotic effects on human prostate cancer cell lines (35). Lignans, recognized as the greatest class of phytoestrogens in the Western diet, have a negative correlation with breast cancer risk in postmenopausal women (36). Apigenin (4',5,7-trihydroxyflavone), a major plant flavone, has shown anticancer properties reducing the risk of certain cancers alone and/or increasing the efficacy of several chemotherapeutic drugs. It could affect several molecular and cellular targets related to a variety of human cancers, in particular, inducing differential effects in causing minimal toxicity to normal cells (37). However, the underlying mechanisms are still unclear. Kang et al. indicated that polyphenols exerted anti-tumor actions as small molecular inhibitors of signaling cascades and src family kinase (38). EGCG induced apoptosis in human acute promyelocytic leukemia NB4 cells and increased the level of $\mathrm{Bcl}-2$-associated $\mathrm{X}$ protein (Bax) protein expression via upregulating Src homology 1 domaincontaining protein tyrosine phosphatase (SHP-1)p38aMAPK- Bax cascade (10). EGCG in combination with Am80 (a synthetic retinoid) synergistically induced apoptosis in human lung cancer cell line PC-9 and up-regulated expressions of growth arrest and DNA damage-inducible gene 153 (GADD153), death receptor 5 , p21waf1 genes by increasing acetylation levels in nonhistone proteins such as p53, $\alpha$-tubulin via down-regulation of histone deacetylase 4, 5, 6 (39). In addition, inflammation is increasingly found to be involved in the development of different types of cancer and the anti-inflammatory property of some polyphenols might provide a therapeutic window for the treatment of cancer. For example, curcumin and rutin reduced tumorassociated inflammation in HPV16-transgenic FVB/ $\mathrm{n}$ mice through inhibition of the expression of cyclooxygenase-2 (COX-2) (40). Curcumin has also been reported to restore the level of tumor suppressor p53 through increasing nuclear factor-erythroid 2 related factor 2 (Nrf2) activation and upregulation of tumor growth factor $\beta$ (TGF- $\beta$ ) and inducible nitric oxide 
synthase (iNOS) in lymphoma bearing mice (41). These indicate that polyphenols have the potential for the development of new strategies for cancer prevention.

\subsection{Dietary polyphenols and neurodegenerative diseases}

The typical diagnosis of AD includes neuropathological lesions, such as amyloid plaques and cerebral amyloid angiopathy, and substantial neuronal loss (42). Resveratrol, which has been found in more than 70 plant species, promotes the non-amyloidogenic cleavage of the amyloid precursor protein, enhances clearance of amyloid beta-peptides, and reduces neuronal damage (43). EGCG, the major component of green tea, exerted a neurorescue effect against functional and neurochemical deficits in a mouse model of Parkinson disease (PD) by regulating the iron-export protein ferroportin in substantia nigra and reducing oxidative stress (44). The flavanone hesperetin, has also been shown to have a protective effect on an early model of PD induced by 6-hydroxydopamine via mitigation of nigral DNA fragmentation as an index of apoptosis and prevention of loss of substantia nigra pars compacta (SNC) dopaminergic neurons (45). Except for the amyloid neurotoxicity, mitochondrial dysfunction and oxidative damage may also play important roles in the slowly progressive neuronal death $(46,47)$. Numerous studies have indicated that dietary polyphenolic compounds exhibited neuroprotective effects through scavenging free radicals and increasing anti-oxidant capacity in vitro and in vivo AD models. Furthermore, they could facilitate the endogenous antioxidant system by stimulating transcription $(48,49)$. Feng et al. reported that grape seed extract (GSE) reduced the concentration of brain 8-isoprostaglandin F2 $\alpha$ and proapoptotic protein c-jun in the brain cortex improving neurofunctional abnormalities caused by hypoxia-ischemia. GSE also reduced brain injury by suppressing lipid peroxidation after reoxygenation in rat pups (50). Long-term consumption of fermented rooibos herbal tea significantly reduced brain edema and neuronal apoptosis by reducing lipid peroxidation levels and increasing total antioxidant capacity in Wistar rats following cerebral ischemia induced by bilateral occlusion of the common carotid arteries (51). Oral consumption of flavonoids may promote neural protection by facilitating the expression of gene products responsible for detoxifying the ischemic microenvironment through both anti-oxidative and antiinflammatory actions. In particular, the transcriptional factor, nuclear factor erythroid 2-related factor 2 (NFE2L2), has emerged as a critical regulator of flavonoid-mediated protection through induction of various cytoprotective genes (52). Some reports have shown that the neuroprotective effects of polyphenols may be associated with activation or inhibition of some cellular signaling pathways involving Sirtuin 1 (SIRT1),
AMPK, etc. Resveratrol is the first polyphenolic compound, which has been shown to activate SIRT1 (53). SIRT1 plays an essential role in the ability of moderate doses of resveratrol to stimulate AMPK and improve mitochondrial function both in vitro and in vivo (54). Wang et al. reported that resveratrol reversed rotenone-induced neurotoxicity through activation of the SIRT/Akt1 signaling pathway and reduction of reactive oxygen species (ROS) production in PC12 cells (55). A recent report showed that strawberry supplementation improved aging-associated impairments in mitochondrial function and biogenesis through increasing the expression of AMPK cascade genes and decreasing intracellular ROS levels and lipid and DNA damage in old rats (56). Studies with lower organisms have also revealed that the increase of AMPK activity could extend lifespan (57).

\section{The potential cellular and molecular mechanism of polyphenols on chronic degenerative diseases}

Molecular mechanisms underlying the potential therapeutic role of polyphenols on CDD have been investigated (Table 3). Here, we will briefly summarize the regulation effects of dietary polyphenols on DNA damage, transcription factors and mRNA expression.

\subsection{Dietary polyphenols inhibit DNA damage}

DNA is easily assaulted by the byproducts of cellular metabolism, background radiation and environmental mutagens. Particularly, DNA double-strand breaks may result in cell death or cancer if improperly repaired. It could be induced by reactive oxygen species, ionizing radiation and some anti-cancer drugs. Polyphenols and flavonoids in artichoke leaf tincture have been shown to inhibit oxidative DNA damage to limit the effects of the atherogenic diet through reducing monocyte chemoattractant protein-1 (MCP-1) expression (58). Seo and Lee demonstrated that antioxidant supplementation including polyphenols, ascorbate and curcumin to lymphocytes inhibited benz[a]anthracene-induced oxidative DNA damage in vitro (59). Resveratrol has also shown the ability to antagonize DNA damage induced by aflatoxin B1 in human lymphocytes (60). Dietary polyphenols such as quercetin, myricetin and catechin could protect HepG2 cells against DNA strand breaks and oxidized pyrimidines induced by $\mathrm{N}$-nitrosodimethylamine, N-nitrosopyrrolidine and benzo(a)pyrene, which are three carcinogenic compounds in the environment (61). Furthermore, the hypermethylation of promoter sequences is the main mechanism of epigenetic inactivation of various genes that are necessary for the accurate regulation of apoptosis, cell cycle or DNA repair. Studies reported that polyphenols (EGCG, dietary black tea and coffee polyphenols, annurca apple polyphenols) have 
Table 3. Molecular mechanism of dietary polyphenols for treatment of CDD

\begin{tabular}{|c|c|c|}
\hline Disease & Action & Signal pathway \\
\hline \multirow[t]{8}{*}{ Cardiovascular disease } & Antiproliferation of HVSMCs & $\downarrow$ AT-1R, $\downarrow$ p-ERK1/2, $\downarrow$ p-P38/MAPK \\
\hline & Antiplatelet aggregation & $\downarrow$ Fibrinogen binding to GPIIb-IIIa receptor; \\
\hline & & $\downarrow$ P-selectin expression \\
\hline & Free radical scavenging & \\
\hline & Anti-inflammation & $\downarrow N F-k B, \uparrow P P A R-\gamma$ and AMPK \\
\hline & Anti-arthrosclerosis & $\downarrow \mathrm{MCP}-1$ \\
\hline & Endothelial NO production & $\downarrow$ Cav-1 mRNA level \\
\hline & Endothelial protection by inhibiting PAI-1 & $\uparrow E C$ p38, $\uparrow \mathrm{ERK} 1 / 2, \uparrow J \mathrm{NK}$ \\
\hline \multirow[t]{5}{*}{ Neurodegenerative disease } & $\begin{array}{l}\text { Clearance of amyloid beta-peptides; Reduction of } \\
\text { oxidative stress and lipid peroxidation; Mitigation of DNA } \\
\text { fragmentation; Prevention of loss of dopaminergic neurons; }\end{array}$ & \\
\hline & Antihypoxia-ischemia & $\downarrow$ brain 8 -isoPGF $2 \alpha, \downarrow$ c-jun \\
\hline & Neuroprotection & 个NFE2L2; Regulation on iron-export protein ferroportin \\
\hline & Relieve neurotoxicity and improve mitochondrial function & $\uparrow$ SIRT/Akt1, $\uparrow$ AMPK \\
\hline & Demethylation activity to improve brain performance & $\downarrow D n m t 3 a$ \\
\hline \multirow[t]{4}{*}{ Cancer } & Anti-tumor actions & $\downarrow$ Src family kinase \\
\hline & Apoptosis induction & $\begin{array}{l}\uparrow \text { Bax, upregualtion of SHP-1-p38MAPK-Bax; } \\
\uparrow \text { GADD153; } \uparrow \text { death receptor } 5 \text {; } \uparrow \text { p21wafl genes; } \uparrow p 53 \text {, } \\
\uparrow N r 2, \uparrow T G F-\beta, \uparrow \text { iNOS } \\
\uparrow \text { SIRT1/Akt }\end{array}$ \\
\hline & Antiproliferation & $\downarrow$ Telomerase mRNA level \\
\hline & Reduced tumor-associated inflammation & $\downarrow \mathrm{COX} 2 ; \downarrow \mathrm{NF}-\mathrm{kB} / \mathrm{p} 65$ activation; $\uparrow \mathrm{PPAR}-\gamma$ \\
\hline
\end{tabular}

HVSMCs: human vascular smooth muscle cells; AT-1R: angiotensin II type 1 receptor; ERK1/2: extracellular regulated protein kinases 1/2; MAPK: mitogen-activated protein kinase; NF-кB: Nuclear transcription factor; PPAR- $\gamma$ : peroxisome proliferators-activated receptors- $\gamma$; AMPK: AMPactivated protein kinase; MCP-1: monocyte chemoattractant protein-1; Cav-1: caveolin-1; EC p38: endothelial cell p38; PAI-1: plasminogen activator inhibitor type-1; JNK: c-Jun N-terminal kinase; 8-isoPGF2 $\alpha$ : 8-iso-prostaglandin F2 $\alpha$; NFE2L2: nuclear factor erythroid 2-related factor 2; SIRT: Sirtuin 1; Dnmt3a: DNA methyltransferase 3a; SHP-1: Src homology 1 domain-containing protein tyrosine phosphatase; GADD153: growth arrest and DNA damage-inducible gene 153; iNOS: inducible nitric oxide synthase; COX2: cyclooxygenase 2.

demethylation activity by inhibiting mammalian DNA methyltransferase 3a (Dnmt3a) to show potential anticancer effects, or improvement of brain performance $(62,63)$.

\subsection{Dietary polyphenols regulate transcription factors}

Nuclear transcription factor NF- $\kappa \mathrm{B}$ regulates the expression of many genes, which encode proteins involved in immune response, inflammatory reaction, apoptosis and neurodegenerative diseases $(64,65)$. Special polyphenols could modulate the expression of genes, which are regulated by NF- $\mathrm{KB}$ at multiple levels. For example, Epicatechin and dimeric procyanidins

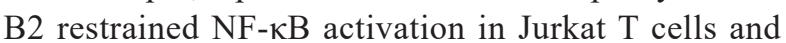
Hodgkin's lymphoma cells by interacting with NF$\kappa \mathrm{B}$ proteins and preventing the binding of NF- $\mathrm{\kappa B}$ to

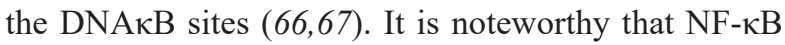
can be constitutively activated in most types of human cancer including breast, colon, skin, lung, esophagus, pancreas, prostate and gliomas and plays a critical role in the regulation of cell survival, proliferation and apoptosis $(68,69)$. A polyphenolic fraction of green tea (GTP) inhibited cell proliferation and induced apoptosis of human osteosarcoma SAOS-2 cells by decreasing nuclear DNA binding of NF- $\kappa \mathrm{B} / \mathrm{p} 65$ and lowering the NF- $\mathrm{kB} / \mathrm{p} 65$ and $\mathrm{p} 50$ levels in the cytoplasm and nucleus (70). Moreover, red wine metabolites may delay the activation of different transcription factors, NF- $\mathrm{KB}$ and activator protein-1 induced by TNF- $\alpha$ to prevent cell adhesion (71). PPAR- $\gamma$ is another nuclear receptor and transcription factor, which is involved in cell control, proliferation and differentiation, exerting anti-inflammatory, anti-cancer and insulin-sensitizing actions. Polyphenols have been reported to regulate the expression of both PPAR- $\gamma$ and NF- $\mathrm{KB}$. Curcuma longa polyphenols have been shown to improve obesity-related metabolic disorders by upregulating the gene expression of PPAR- $\gamma$ and decreasing the production of pro-inflammatory molecules such as NF-kB, MCP-1, interleukin 6 (IL-6), etc. in 3T3-L1 adipose cells exposed to hydrogen peroxide $\left(\mathrm{H}_{2} \mathrm{O}_{2}\right)$ mediated oxidative stress (72). Choi et al. has also shown that galangin exerts anti-inflammatory effects by inhibiting NF- $\mathrm{\kappa B}$ activity and increasing expression and transcriptional activity of PPAR- $\gamma$ in polyinosinicpolycytidylic acid (poly(I:C)) (a viral mimic dsRNA analog)-stimulated microglia (73).

\subsection{Dietary polyphenols regulate $m R N A$ expression}

Telomerase, a specialized reverse transcriptase, has an important role in cell fate, for example, sustaining cell proliferation resulting in tumorigenesis and mutagenesis. The activity of telomerase correlates with the degree of malignancy and senescence (74). Tea 
polyphenols of EGCG and epigallocatechin (EGC) showed repression activities on carcinogenesis by inhibiting mRNA expression of human telomerase reverse transcriptase (hTERT) in human lung carcinoma H1299 cells, human oral cancer OECM-1 cells and tongue squamous-cell carcinoma cells (SAS) (75). In the cardiovascular system, caveolin-1 (Cav-1), a negative regulator of endothelial nitric oxide synthase, influences various aspects of cardiovascular function. Green tea polyphenols could down-regulate mRNA levels of Cav-1 in bovine aortic endothelial cells timeand dose-dependently via activating ERK1/2 and inhibiting p38MAPK signaling (76). Furthermore, there is a positive association between plasminogen activator inhibitor type-1 (PAI-1) and the development and progression of CVD. Catechin and quercetin showed cardiovascular protection through down-regulating the PAI-1 mRNA level in endothelial cells via activating ERK1/2 and c-Jun N-terminal kinase (JNK) (77). A resveratrol-rich grape has also been reported to reduce low-density lipoprotein (LDL)-cholesterol, oxidized LDL and apolipoprotein B (ApoB) and improve the inflammatory and fibrinolytic status in patients undergoing primary prevention of CVD through decreasing thrombogenic PAI-1 by modulating six key inflammation-related transcription factors involved in inflammation and cell migration (78).

\section{The polyphenols-gut microbiota interactions}

There exists a complex microbial ecosystem comprising considerable metabolic versatility in the human intestinal tract (79). Reports have shown that dietary polyphenols could affect the composition and function of gut microbiota involved in the maintenance of gastrointestinal health. The equilibrium of the gut microbiota in return keeps the bioavailability and physiological activity of dietary polyphenols. The growth of certain pathogenic bacteria such as Clostridium perfringens, Clostridium difficile and Bacteroides spp. were significantly repressed by tea phenolics and their derivatives, while the growth of commensal anaerobes like Clostridium spp., Bifidobacterium spp. and probiotics such as Lactobacillus $s p$. were not affected. This suggested that different strains of intestinal bacteria had different sensitivity to tea phenolics and their metabolites (80). Moreover, consistent evidence from Shanthi et al. indicates that polyphenols have potential to alter gut microecology and confer positive gut health benefits by regulating the total number of beneficial microflora in the gut (81). There is a growing body of evidence that gut microbiota contributes to colon tumorigenesis. Polyphenols can inhibit the development of colon tumorigenesis through affecting the composition and activity of the gut microbiota and anti-inflammation. On the other hand, gut microbiota are involved in the metabolism of polyphenols and convert dietary polyphenols into active and bioavailable metabolites. As gut microbiota vary among individuals, variations in gut microbiota can affect polyphenol activity by producing metabolites with different physiological significance (82). For example, the soy isoflavone daidzein is microbially biotransformed to equol, which has a more potent estrogenic action than the precursor itself. Being overweight or obese induced the gut microbial environment alteration and contributed to being incapable of metabolizing the soy isoflavone daidzine to O-desmethylangolensin (ODMA) in periand post-menopausal women (83). What's more, human intestinal bacteria could rapidly degrade cyranidin 3-glucoside (C3G, one of the major dietary anthocyanins) to phenolic acids in vitro. However, higher concentrations of phenolic acids were observed in the presence of intestinal bacteria, while these products appeared at considerably lower levels in the absence of bacteria (84).

\section{The potential danger of dietary polyphenols on human health}

Many types of polyphenols are considered to be traditional herbal medicines and safety drug candidates. However, it is noteworthy that some actions of individual dietary polyphenols may have a potential danger to human health. Tannins, catechins, flavones and isoflavones have been considered as topo poisons, which could cause high numbers of broken and recombined chromosomes and chromosome aberrations in treated cells $(85,86)$. Green tea polyphenols (GTPs) showed toxicity at high doses presumably due to prooxidative properties in experimental rodents, such as hepatotoxicity, GTP-deteriorated dextran sodium sulfate (DSS)-induced intestinal inflammation and carcinogenesis, 1\% GTPs induced nephrotoxicity in colitis mode mice, etc. (87). Quercetin transformed into an ortho-quinone after scavenging free radicals to decrease the level of many anti-oxidative cellular substances such as glutathione (GSH), and total antioxidative capacity (88). Biso et al. reported that quercetin, quercetin-3-O- $\alpha$-1-rhamnoside (quercitrin), myricetin and gallic acid derivatives showed mutagenic potential for peripheral blood cells by micronucleus test (Ames test), while they didn't show genotoxic activity in the mouse cells (90). Another study observed the mutagenicity of ten flavonoids, results showed that some flavonoids such as galangin, kaemferol and quercetin could be biotransformed into more genotoxic metabolites and showed signs of mutagenicity in Ames test (90). The structural requirements for mutagenicity is a flavonoid ring structure with a free hydroxyl group at the 3-position, a double bond at the 2,3-position and a keto-group at the 4-position allowing the proton of the 3 -hydroxyl group to tautomerize to 
a 3-keto moiety. When free hydroxyl groups are not present in the B-ring, a metabolic activating system is required for the formation of a mutagenic compound (91). In addition, flavonoids in propolis induced both mutagenic and antimutagenic effects depending on its concentration (92). The result is consistent with the study that green propolis caused an increase in the damage to DNA in the peripheral blood cells of mice using micronucleus and single-cell gel electrophoresis assays (93). Comparable to green propolis, the extracts of baccharis dracunculifolia showed genotoxic and mutagenic effects by increasing the DNA damage in blood and liver tissues and the frequency of micronuclei in bone marrow in mice (94). Cecropia pachystachya, which contains chlorogenic acid, isoorientin, orientin, and isovitexin, were shown to be genotoxic to brain tissue because of the ability to cross the blood-brain barrier and act on the central nervous system and had mutagenic effects at higher doses (95).

\section{Prospects of dietary polyphenols in treatment of CDD}

Recent investigations of the pharmacological actions of dietary polyphenols have showed that they have neuroprotection, free radical scavenging, antioxidation, reduction of LDL-cholesterol and ApoB, antiinflammation, carcinogenesis repression, inhibition on DNA damage, etc. which promised strong potential for treatment of $\mathrm{AD}, \mathrm{PD}, \mathrm{CVD}$, and cancers. However, more research will be needed to further investigate the structure-activity relationship of polyphenols molecules and the mechanism of biological effects including beneficial or deleterious effects before treating dietary polyphenols as therapeutic agents.

\section{Acknowledgements}

This work was supported by Research Training Project of Taishan College, Shandong University.

\section{References}

1. Boudet AM. Evolution and current status of research in phenolic compounds. Phytochemistry. 2007; 68:27222735.

2. Wang K, Jiang S, Pu T, Fan L, Su F, Ye M. Antifungal activity of phenolic monoterpenes and structure-related compounds against plant pathogenic fungi. Nat Prod Res. 2018; 15:1-8.

3. Petti S, Scully C. Polyphenols, oral health and disease: a review. J Dent. 2009; 37:413-423.

4. Adefegha SA. Functional foods and nutraceuticals as dietary intervention in chronic diseases; novel perspectives for health promotion and disease prevention. J Diet Suppl. 2017; 27:1-33.

5. Tomás-Barberán FA, Andrés-Lacueva C. Polyphenols and health: current state and progress. J Agr Food Chem. 2012; 60:8773-8775.
6. Visioli F, Davalos A. Polyphenols and cardiovascular disease: a critical summary of the evidence. Mini-Rev Med Chem. 2011; 11:1186-1190.

7. Féart C, Samieri C, Barberger-Gateau P. Mediterranean diet and cognitive function in older adults. Curr Opin Clin Nutr Metab Care. 2010; 13:14-18.

8. Leifert WR, Abeywardena MY. Cardioprotective actions of grape polyphenols. Nutr Res. 2008; 28:729-737.

9. Rodrigo R, Miranda A, Vergara L. Modulation of endogenous antioxidant system by wine polyphenols in human disease. Clin Chim Acta. 2011; 412:410-424.

10. Gan L, Zhong L, Shan Z, Xiao C, Xu T, Song H, Li L, Yang R, Liu B. Epigallocatechin-3-gallate induces apoptosis in acute promyelocytic leukemia cells via a SHP-1-p38 $\alpha$ MAPK-Bax cascade. Oncol Lett. 2017; 14:6314-6320.

11. Coniglio MS, Busto VD, González PS, Medina MI, Milrad S, Agostini E. Application of Brassica napus hairy root cultures for phenol removal from aqueous solutions. Chemosphere. 2008; 72:1035-1042.

12. Belcavello L, Vencioneck Dutra JC, de Freitas JV, Aranha IP, do Carmo Pimentel, Batitucci M. Mutagenicity of ipriflavone in vivo and in vitro. Food Chem Toxicol. 2012; 50:996-1000.

13. Macáková K, Mladěnka $P$, Filipský $\mathrm{T}$, Ríha M, Jahodář L, Trejtnar F, Bovicelli P, Proietti Silvestri I, Hrdina R, Saso L. Iron reduction potentiates hydroxyl radical formation only in flavonols. Food Chem. 2012; 135:2584-2592.

14. Fraga CG, Galleano M, Verstraeten SV, Oteiza PI. Basic biochemical mechanisms behind the health benefits of polyphenols. Mol Aspects Med. 2010; 31:435-445.

15. Davin LB, Lewis NG. An historical perspective on lignan biosynthesis: monolignol, allylphenol and hydroxycinnamic acid coupling and downstream metabolism. Phytochem Rev. 2003; 2: 257-288.

16. Rodríguez-Cabo T, Rodríguez I, Ramil M, Cela R. Comprehensive evaluation of the photo-transformation routes of trans-resveratrol. J Chromatogr A. 2015; 1410:129-139.

17. Gaya P, Medina M, Sánchez-Jiménez A, Landete JM. Phytoestrogen metabolism by adult human gut microbiota. Molecules. 2016; 21:pii: E1034.

18. Bhattacharjee R, Devi A, Mishra S. Molecular docking and molecular dynamics studies reveal structural basis of inhibition and selectivity of inhibitors EGCG and OSU03012 toward glucose regulated protein-78 (GRP78) overexpressed in glioblastoma. J Mol Model. 2015; 21:272.

19. Berindan-Neagoe I, Braicu C, Tudoran O, Balacescu O, Irimie A. Early apoptosis signals induced by a low dose of epigallocatechin 3-gallate interfere with apoptotic and cell death pathways. J Nanosci Nanotechno. 2012; 12:21132119.

20. Xiao J, Kai G, Yang F, Liu C, Xu X, Yamamoto K. Molecular structure-affinity relationship of natural polyphenols for bovine $\gamma$-globulin. Mol Nutr Food Res. 2011; 55(Suppl 1):S86-92.

21. Heim KE, Tagliaferro AR, Bobilya DJ. Flavonoid antioxidants: chemistry, metabolism and structure-activity relationships. J Nutr Biochem. 2002; 13:572-584.

22. Chakraborty S, Biswas PK. Elucidation of the mechanistic pathways of the hydroxyl radical scavenging reaction by daidzein using hybrid QM/MM dynamics. J Phys Chem A. 2012; 116:8775-8785

23. Ali HM, Almagribi W, Al-Rashidi MN. Antiradical and 
reductant activities of anthocyanidins and anthocyanins, structure-activity relationship and synthesis. Food Chem. 2016; 194:1275-1282.

24. Saragusti AC, Ortega MG, Cabrera JL, Estrin DA, Marti MA, Chiabrando GA. Inhibitory effect of quercetin on matrix metalloproteinase 9 activity Molecular mechanism and structure-activity relationship of the flavonoid-enzyme interaction. Eur J Pharmacol. 2010; 644:138-145.

25. Cai YZ, Sun M, Xing J, Luo Q, Corke H. Structure-radical scavenging activity relationships of phenolic compounds from traditional Chinese medicinal plants. Life Sci. 2006; 78:2872-2888

26. Vasanthi HR, Shrishrimal N, Das DK. Phytochemicals from plants to combat cardiovascular disease. Curr Med Chem. 2012; 19:2242-2251.

27. Yang L, Zhang Y, Zhu M, Zhang Q, Wang X, Wang Y, Zhang J, Li J, Yang L, Liu J, Liu F, Yang Y, Kang L, Shen Y, Qi Z. Resveratrol attenuates myocardial ischemia/ reperfusion injury through up-regulation of vascular endothelial growth factor B. Free Radic Biol Med. 2016; 101:1-9.

28. Zhan XL, Yang XH, Gu YH, Guo LL, Jin HM. Epigallocatechin gallate protects against homocysteineinduced vascular smooth muscle cell proliferation. Mol Cell Biochem. 2018; 439:131-140.

29. Santhakumar AB, Bulmer AC, Singh I. A review of the mechanisms and effectiveness of dietary polyphenols in reducing oxidative stress and thrombotic risk. J Hum Nutr Diet. 2014; 27:1-21.

30. Khan H, Jawad M, Kamal MA, Baldi A, Xiao J, Nabavi SM, Daglia M. Evidence and prospective of plant derived flavonoids as antiplatelet agents: Strong candidates to be drugs of future. Food Chem Toxicol. 2018; 119:355-367.

31. McEwen BJ. The influence of diet and nutrients on platelet function. Semin Thromb Hemost. 2014; 40:214226.

32. Sears B, Ricordi C. Role of fatty acids and polyphenols in inflammatory gene transcription and their impact on obesity, metabolic syndrome and diabetes. Eur Rev Med Pharmacol Sci. 2012; 16:1137-1154.

33. Singhal K, Raj N, Gupta K, Singh S. Probable benefits of green tea with genetic implications. J Oral Maxillofac Pathol. 2017; 21:107-114.

34. Hessien M, El-Gendy S, Donia T, Sikkena MA. Growth inhibition of human non-small lung cancer cells h460 by green tea and ginger polyphenols. Anticancer Agents Med Chem. 2012; 12:383-390.

35. Bennani H, Drissi A, Giton F, Kheuang L, Fiet J, Adlouni A. Antiproliferative effect of polyphenols and sterols of virgin argan oil on human prostate cancer cell lines. Cancer Detect Prev. 2007; 31:64-69.

36. Buck K, Zaineddin AK, Vrieling A, Linseisen J, ChangClaude J.. Meta-analyses of lignans and enterolignans in relation to breast cancer risk. Am J Clin Nutr. 2010; 92:141-153.

37. Shankar E, Goel A, Gupta K, Gupta S. Plant flavone apigenin: an emerging anticancer agent. Curr Pharmacol Rep. 2017; 3:423-446.

38. Kang NJ, Shin SH, Lee HJ, Lee KW. Polyphenols as small molecular inhibitors of signaling cascades in carcinogenesis. Pharmacol Ther. 2011; 130:310-324.

39. Oya Y, Mondal A, Rawangkan A, Umsumarng S, Iida K, Watanabe T, Kanno M, Suzuki K, Li Z, Kagechika H, Shudo K, Fujiki H, Suganuma M. Down-regulation of histone deacetylase $4,-5$ and -6 as a mechanism of synergistic enhancement of apoptosis in human lung cancer cells treated with the combination of a synthetic retinoid, Am80 and green tea catechin. J Nutr Biochem. 2017; 42:7-16.

40. Das L, Vinayak M. Long term effect of curcumin in restoration of tumour suppressor p53 and phase-II antioxidant enzymes via activation of Nrf2 signalling and modulation of inflammation in prevention of cancer. PLoS One. 2015; 10:e124000.

41. Moutinho MSS, Aragão S, Carmo D, Casaca F, Silva S, Ribeiro J, Sousa H, Pires I, Queiroga F, Colaço B, Medeiros R, Oliveira PA, Lopes C, Bastos MMSM, DA Costa RMG. Curcumin and Rutin down-regulate cyclooxygenase-2 and reduce tumor-associated inflammation in HPV16-Transgenic Mice. Anticancer Res. 2018; 38:1461-1466.

42. Serrano-Pozo A, Frosch MP, Masliah E, Hyman BT. Neuropathological alterations in Alzheimer disease. Cold Spring Harb Perspect Med. 2011; 1:a006189.

43. Li F, Gong Q, Dong H, Shi J. Resveratrol, a neuroprotective supplement for Alzheimer's disease. Curr Pharm Design. 2012; 18:27-33.

44. Xu Q, Langley M, Kanthasamy AG, Reddy MB. Epigallocatechin gallate has a neurorescue effect in a mouse model of Parkinson disease. J Nutr. 2017; 147:1926-1931.

45. Kiasalari Z, Khalili M, Baluchnejadmojarad T, Roghani M. Protective effect of oral hesperetin against unilateral striatal 6-hydroxydopamine damage in the rat. Neurochem Res. 2016; 41:1065-1072.

46. Schapira AH. Mitochondrial dysfunction in neurodegenerative diseases. Neurochem Res. 2008; 33:2502-2509.

47. Trushina E, McMurray CT. Oxidative stress and mitochondrial dysfunction in neurodegenerative diseases. Neuroscience. 2007; 145:1233-1248.

48. Singh M, Arseneault M, Sanderson T, Murthy V, Ramassamy C. Challenges for research on polyphenols from foods in Alzheimer's disease: bioavailability, metabolism, and cellular and molecular mechanisms. J Agr Food Chem. 2008; 56:4855-4873.

49. Choi DY, Lee YJ, Hong JT, Lee HJ. Antioxidant properties of natural polyphenols and their therapeutic potentials for Alzheimer's disease. Brain Res Bull. 2012; 87:144-153.

50. Feng Y, Liu YM, Leblanc MH, Bhatt AJ, Rhodes PG. Grape seed extract given three hours after injury suppresses lipid peroxidation and reduces hypoxicischemic brain injury in neonatal rats. Pediatr Res. 2007; 61:295-300.

51. Akinrinmade O, Omoruyi S, Dietrich D, Ekpo O. Longterm consumption of fermented rooibos herbal tea offers neuroprotection against ischemic brain injury in rats. Acta Neurobiol Exp (Wars). 2017; 77:94-105.

52. Leonardo CC, Doré S. Dietary flavonoids are neuroprotective through Nrf2-coordinated induction of endogenous cytoprotective proteins. Nutr Neurosci. 2011; 14:226-236.

53. Baur JA, Pearson KJ, Price NL, et al. Resveratrol improves health and survival of mice on a high-calorie diet. Nature. 2006; 444:337-342.

54. Price NL, Gomes PG, Ling AJ, et al. SIRT1 is required for AMPK activation and the beneficial effects of resveratrol on mitochondrial function. Cell Metab. 2012; 15:675-690.

55. Wang H, Dong X, Liu Z, et al. Resveratrol suppresses 
rotenone-induced neurotoxicity through activation of SIRT1/Akt1 signaling pathway. Anat Rec (Hoboken). 2018; 301:1115-1125.

56. Giampieri F, Alvarez-Suarez JM, Cordero MD, Gasparrini M, Forbes-Hernandez TY, Afrin S, Santos-Buelga C, González-Paramás AM, Astolfi P, Rubini C, Zizzi A, Tulipani S, Quiles JL, Mezzetti B, Battino M. Strawberry consumption improves aging-associated impairments, mitochondrial biogenesis and functionality through the AMP-activated protein kinase signaling cascade. Food Chem. 2017; 234:464-471.

57. Salminen A, Kaarniranta K. AMP-activated protein kinase (AMPK) controls the aging process via an integrated signaling network. Ageing Res Rev. 2012; 11:230-241.

58. Bogavac-Stanojevic N, Kotur Stevuljevic J, Cerne D, Zupan J, Marc J, Vujic Z, Crevar-Sakac M, Sopic M, Munjas J, Radenkovic M, Jelic-lvanovic Z. The role of artichoke leaf tincture (Cynara scolymus) in the suppression of DNA damage and atherosclerosis in rats fed an atherogenic diet. Pharm Biol. 2018; 56:138-144.

59. Seo YN, Lee MY. Inhibitory effect of antioxidants on the benz[a]anthracene- induced oxidative DNA damage in lymphocytes. J Environ Biol. 2011; 32:7-10.

60. Türkez H, Sisman T. The genoprotective activity of resveratrol on aflatoxin B1-induced DNA damage in human lymphocytes in vitro. Toxicol Ind Health. 2012; 28:474-480.

61. Delgado ME, Haza AI, Arranz N, Garcia A, Morales P. Dietary polyphenols protect against $\mathrm{N}$-nitrosamines and benzo(a)pyrene-induced DNA damage (strand breaks and oxidized purines/pyrimidines) in HepG2 human hepatoma cells. Eur J Nutr. 2008; 47:479-490.

62. Rajavelu A, Tulyasheva Z, Jaiswal R, Jeltsch A, Kuhnert N. The inhibition of the mammalian DNA methyltransferase 3a (Dnmt 3a) by dietary black tea and coffee polyphenols. BMC Biochem. 2011; 12:16.

63. Fini L, Selgrad M, Fogliano V, Graziani G, Romano M, Hotchkiss E, Daoud YA, De Vol EB, Boland CR, Ricciardiello L. Annurca apple polyphenols have potent demethylation activity and can reactivate silenced tumor suppressor genes in colorectal cancer cells. J Nutr. 2007; 137:2622-2628.

64. Radhakrishnan SK, Kamalakaran S. Pro-apoptotic role of NF-kappaB: implications for cancer therapy. Biochim Biophys Acta. 2006; 1766:53-62.

65. Perkins ND. Integrating cell-signalling pathways with NFkappaB and IKK function. Nat Rev Mol Cell Biol. 2007; 8:49-62.

66. Mackenzie GG, Oteiza PI. Modulation of transcription factor NF-kappaB in Hodgkin's lymphoma cell lines: effect of (-)-epicatechin. Free Radic Res. 2006; 40:10861094.

67. Mackenzie GG, Adamo AM, Decker NP, Oteiza PI. Dimeric procyanidin B2 inhibits constitutively active NFkappaB in Hodgkin's lymphoma cells independently of the presence of IkappaB mutations. Biochem Pharmacol. 2008; 75:1461-1471.

68. Li F, Zhang J, Arfuso F, Chinnathambi A, Zayed ME, Alharbi SA, Kumar AP, Ahn KS, Sethi G. NF- $\kappa$ B in cancer therapy. Arch Toxicol. 2015; 89:711-731.

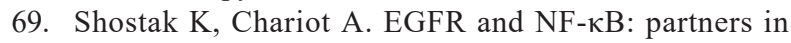
cancer. Trends Mol Med. 2015; 21:385-393.

70. Hafeez BB, Ahmed S, Wang N, Gupta S, Zhang A, Haqqi TM. Green tea polyphenols-induced apoptosis in human osteosarcoma SAOS-2 cells involves a caspase-dependent mechanism with downregulation of nuclear factor- $\kappa \mathrm{B}$. Toxicol Appl Pharmacol. 2006; 216:11-19.

71. Canali R, Comitato R, Ambra R, Virgili F. Red wine metabolites modulate NF-kappaB, activator protein-1 and cAMP response element-binding proteins in human endothelial cells. Brit J Nutr. 2010; 103:807-814.

72. Septembre-Malaterre A, Le Sage F, Hatia S, Catan A, Janci L, Gonthier MP. Curcuma longa polyphenols improve insulin-mediated lipid accumulation and attenuate proinflammatory response of 3T3-L1 adipose cells during oxidative stress through regulation of key adipokines and antioxidant enzymes. Biofactors. 2016; 42:418-430.

73. Choi MJ, Park JS, Park JE, Kim HS, Kim HS. Galangin suppresses pro-inflammatory gene expression in polyinosinic-polycytidylic acid-stimulated microglial Cells. Biomol Ther (Seoul). 2017; 25:641-647.

74. $\mathrm{Xu} \mathrm{Y,} \mathrm{He} \mathrm{K,} \mathrm{Goldkorn} \mathrm{A.} \mathrm{Telomerase} \mathrm{targeted} \mathrm{therapy} \mathrm{in}$ cancer and cancer stem cells. Clin Adv Hematol Oncol. 2011; 9:442-455.

75. Lin SC, Li WC, Shin JW, Hong KF, Pan YR, Lin JJ. The tea polyphenols EGCG and EGC repress mRNA expression of human telomerase reverse transcriptase (hTERT) in carcinoma cells. Cancer Lett. 2006; 23:8088 .

76. Li Y, Ying C, Zuo X, Yi H, Yi W, Meng Y, Ikeda K, Ye X, Yamori Y, Sun X. Green tea polyphenols down-regulate caveolin-1 expression via ERK1/2 and p38MAPK in endothelial cells. J Nutr Biochem. 2009; 20:1021-1027.

77. Pasten C, Olave NC, Zhou L, Tabengwa EM, Wolkowicz PE, Grenett HE. Polyphenols downregulate PAI-1 gene expression in cultured human coronary artery endothelial cells: Molecular contributor to cardiovascular protection. Thromb Res. 2007; 121:59-65.

78. Wang S, Moustaid-Moussa N, Chen L, Mo H, Shastri A, Su R, Bapat P, Kwun I, Shen CL. Novel insights of dietary polyphenols and obesity. J Nutr Biochem. 2014; 25:1-18.

79. Qin J, Li R, Raes J, et al. A human gut microbial gene catalogue established by metagenomic sequencing. Nature. 2010; 464:59-65.

80. Lee HC, Jenner AM, Low CS, Lee YK. Effect of tea phenolics and their aromatic fecal bacterial metabolites on intestinal microbiota. Res Microbiol. 2006; 157:876-884.

81. Parkar SG, Stevenson DE, Skinner MA. The potential influence of fruit polyphenols on colonic microflora and human gut health. Int J Food Microbiol. 2008; 124:295298.

82. Tomás-Barberán FA, Selma MV, Espín JC. Interactions of gut microbiota with dietary polyphenols and consequences to human health. Curr Opin Clin Nutr Metab Care. 2016; 19:471-476.

83. Miller LM, Lampe JW, Newton KM, Gundersen G, Fuller S, Reed SD, Frankenfeld CL. Being overweight or obese is associated with harboring a gut microbial community not capable of metabolizing the soy isoflavone daidzein to O-desmethylangolensin in peri- and post-menopausal women. Maturitas. 2017; 99:37-42.

84. Hanske L, Engst W, Loh G, Sczesny S, Blaut M, Braune A. Contribution of gut bacteria to the metabolism of cyanidin 3 -glucoside in human microbiota-associated rats. $\mathrm{Br} \mathrm{J}$ Nutr. 2013; 10:1433-1441.

85. Cantero G, Campanella C, Mateos S, Cortés F. Topoisomerase II inhibition and high yield of endoreduplication induced by the flavonoids luteolin and quercetin. Mutagenesis. 2006; 21:321-325. 
86. Zhoum N, Yanm Y, Lim W, Wangm Y, Zhengm L, Hanm S, Yanm Y, Lim Y. Genistein inhibition of topoisomerase IIalpha expression participated by $\mathrm{Sp} 1$ and $\mathrm{Sp} 3$ in HeLa cells. Int J Mol Sci. 2009; 10:3255-3268.

87. Murakami A. Dose-dependent functionality and toxicity of green tea polyphenols in experimental rodents. Arch Biochem Biophys. 2014; 557:3-10.

88. Rpbaszkiewicz A, Balcerczyk A, Bartosz G. Antioxidative and prooxidative effects of quercetin on A549 cells. Cell Biol Int. 2007; 31:1245-1250.

89. Biso FI, Rodrigues CM, Rinaldo D, Reis MB, Bernardi CC, de Mattos JC, Caldeira-de-Araújo A, Vilegas W, Cólus IM, Varanda EA. Assessment of DNA damage induced by extracts fractions and isolated compounds of Davilla nitida and Davilla elliptica (Dilleniaceae). Mutat Res. 2010; 702:92-99.

90. Resende FA, Vilegas W, Dos Santos LC, Varanda EA. Mutagenicity of flavonoids assayed by bacterial reverse mutation (Ames) test. Molecules. 2012; 17:5255-5268.

91. MacGregor JT, Jurd L. Mutagenicity of plant flavonoids: structural requirements for mutagenic activity in Salmonella typhimurium. Mutat Res. 1978; 54:297-309.
92. Tavares DC, Mazzaron Barcelos GR, Silva LF, Chacon Tonin CC, Bastos JK. Propolis-induced genotoxicity and antigenotoxicity in Chinese hamster ovary cells. Toxicol in Vitro. 2006; 20:1154-1158.

93. Pereira AD, de Andrade SF, de Oliveira-Swerts MS, Maistro EL. First in vivo evaluation of the mutagenic effect of Brazilian green propolis by comet assay and micronucleus test. Food Chem Toxicol. 2008; 46:25802584.

94. Rodrigues CR, Dias JH, Semedo JG, da Silva J, Ferraz AB, Picada JN. Mutagenic and genotoxic effects of Baccharis dracunculifolia (D.C.). J Ethnopharmacol. 2009; 124:321-324.

95. Mendonça ED, da Silva J, Dos Santos MS, Carvalho P, Papke DK, Ortmann CF, Picada JN, Reginatto FH, de Barros Falcão Ferraz A. Genotoxic, mutagenic and antigenotoxic effects of Cecropia pachystachya Trécul aqueous extract using in vivo and in vitro assays. J Ethnopharmacol. 2016; 193:214-220.

(Received July 23, 2018; Revised November 8, 2018; Accepted December 9, 2018) 